\title{
Service excellence in health care
}

\author{
Brian Edwards
}

The first priority for any health care system must be clinical excellence. High quality training, regular peer review, and an effective system of clinical audit will help secure this. Clinical excellence must be related to a concept of outcomes and health gain. We achieve clinical excellence more often and more consistently in the NHS than in almost any other system of health care in the world. We should take pride in this.

However, this clinical excellence is often tarnished from the patient's perspective by a poor overall experience: long waits, lack of privacy, little attempt to personalise care for them as individuals, and poor communication between professionals and patients. Really first class health care combines professional excellence with superb personal service.

This is not the place to rehearse the economics of the NHS except in one regard. The sort of open ended health insurance policy the NHS offers the people of this country works only with a process of rationing. Skilled, caring choices they may be, but the excercise of rationing can, and often does, result in an unhealthy organisational and professional arrogance that is the very antithesis of personal service. The great danger to public service organisations is that they become focused around the needs of those who have the tough job of rationing, rather than around the needs of those who receive the service.

These attitudes have led in the NHS to:

- Block booking of outpatient clinics to conserve the time of the professional staff at almost any cost to patients

- Routine late cancellation of admission to hospital for cold surgery, which is justified on grounds of efficiency

- Long waits in accident and emergency departments and sometimes in the general practitioner's surgery

- A position where a private Marplan poll showed that $49 \%$ of people we serve say they were treated as just another patient with no special or personal recognition.

If we want to preserve a free and comprehensive NHS in the public service we have to do better. That is what this conference is about.

\section{Doing better by patients}

The patient's charter is interesting in that for the first time up front service promises (sometimes stretching to guarantees) are made to the people we serve, in a manner which enables them to judge whether we have lived up to our own standards. Some will say that health is different, that it is much easier to judge service industries like British Rail - for example. Could I get a seat? Was the carriage clean and the buffet car open? Did the train arrive on time?

Also, it is easy to promise but not so easy to deliver. Health is different, setting explicit standards is more difficult, but it can be done. Patients may not be able to judge whether their surgery was performed just competently, or superbly. They may not even realise when it was done badly. They can tell you how long they had to wait for admission. They can tell you how many times their planned admission was cancelled. They know whether they received imediate clinical assessment in the accident and emergency department and how long they then waited for treatment.

Although the patient's charter emanates from John Major, I am assuming that the type of service improvements it seeks will be common ground between the political parties. They certainly ought to be common ground between the professionals providing health care and those trying to manage health organisations. Service standards are not a frill, an extravagance. Patients' overall experience with their health service must impact on the quality of the outcome. Psychiatrists, geriatricians, and paediatricians have known this for years; we just need to persuade the rest of the clinical community. We need to persuade them because we have to invest in service excellence as well as clinical excellence.

\section{Volume versus quality}

The really tough issue is usually presented as the tension between volume and quality.

"Of course I can spend more time with individual patients in my surgery or clinic and give them all a booked appointment if I see fewer." (Or appoint 60000 more consultants!)

"Of course I could reduce trolley waits in accident and emergency if you will accept an increase in waiting lists."

Neither is true and both represent a defensive response to a reasonable challenge. Before confronting the volume versus quality choice in outpatients (and achieve the 30 minute target required by the patient's charter) the following could be attempted.

- Start the clinic on time more often

- Tackle "non-attendance" rates (which often reach $20 \%$ in some specialties).

Overbooking to compensate for high non- 
attendance rates makes clinics even more chaotic and does nothing to deal with the root cause, which are usually:

- No patient choice of date or time for appointments

- Difficulties for patients in informing the clinic they cannot attend

- A belief that clinics are so chaotic that there is no need to keep to time (in practice, many patients arrive far too early anyway).

Potential solutions include:

- Telephoning the patient a few days before the clinic (or a visit by the community nurse); this represents a good return on investment, particularly if concentrated on those clinics experiencing the highest nonattendance rates. (Some hospitals have reduced these to $2 \%$ or less)

- Freephones or answering machines to facilitate changes initiated by patients

- Discussion between consultants, general practitioners, and managers about clinical protocols for referral and frequency of repeat attendances

- Shaping the booking pattern of the clinic more closely to the natural pace of the individual professional.

Once all four solutions have been worked on the problem will have changed in shape and size. If a choice between sustaining a reasonable standard of service and volume still exists - always take the quality option. The other route is a slippery slope to decay and decline.

Cancelling a hospital admission at short notice once in the face of a real emergency is understandable. Doing it two, three, or four times to the same patient is sloppy clinical practice and ineffective hospital management. "Specialing" patients who have had one bad experience produces a simple operational answer. On the second occasion protect and shepherd the patient through the system, as British Airways do with children.

\section{Communication between professionals and patients}

The greatest challenge is in professionalpatient communication. "Tell us more" is the recurrent message from patients about both general practice and hospital medicine. A recent survey showed that $21 \%$ of patients had

\section{Three ways of improving doctor-} patient communication

(1) In an elderly ward - relatives and patients book conversations with the doctor or named nurse, or both. The process of booking seems to give the conversation more status and is perhaps prepared for far better by professional staff

(2) The advice on discharge from hospital is produced on videotape so that the patients can replay it at home (audio tapes work as well). Patients are enthusiastic, lawyers are very uncomfortable with it - it must be right

(3) The general practitioner's referral letter indicates whether an interpreter is needed so that one is waiting, prebooked, at the clinic visit no information or advice about further treatment or management on discharge from hospital. A Marplan poll conducted for Trent Regional Health Authority in October 1991 showed that $28 \%$ of those interviewed did not consider they had received sufficient information about their treatment.

The box shows three simple ideas gleaned from my recent visits in the region.

Also, patient held records are commonplace in child health and obstetrics, but they can be extended to other specialties and linked to general practice. This must be one of the developing trends in professional patient communication.

\section{Working towards the future}

The first edition of the patient's charter has a hospital flavour; we are now working with the professionals in primary care to generate standards in that sector. Together we will find some that blend clinical and service excellence.

A sign of operational unresponsiveness is our attitude to complaints. If they go up, alarm bells ring. When complaints go up in future, I want to say well done to all concerned. We need to regard complaints not as threats but very valuable assets. They tell us how to improve the service. Hunting for faults requires a major shift in culture.

My team and I learnt a lot from our three month experience of helping to implement the patient's charter.

- Clear service standards whose achievement can be measured by both staff and patients are powerful tools in the drive to improve service, provided there are not too many. The impact is blunted with every one added to the list

- Standard setting works best when there is a clear, unambiguous alignment between the service standard to be secured and clinical excellence. So start with them

- The real quality challenge is about consistency. Short term impact on problems are not difficult to achieve. The real challenge is securing the standard, day in, day out, in Dorset, Grimsby, inner cities, and rural communities. That is the sign of a really excellent service organisation

- Hearts and minds are vital to success, but quality evaporates unless firmly underpinned by culture and measuring devices. Progress needs to be monitored

- Some of the service standards need to be as firmly rooted in the organisation as professional standards. Cancelling surgery at short notice, twice for the same patient, should be as unthinkable as not scrubbing up before operating

- System faults are a major problem for the NHS. Much can be learnt from Japanese industry. How many patients experience a fault due to poor internal coordination or communication? The truth is the figure is unknown - probably more than $50 \%$. The system is not designed in a way that has the patient as its exclusive focus

- For every problem somebody in the NHS 
has already found a solution. The richness of ideas and innovative spirit in the health service is amazing for a public sector organisation. In terms of service standards the NHS is bubbling with ideas.

How can progress be achieved?

(1) Secure clinical excellence as the foundation stone; make clinical audit really work; maintain teaching standards; drive the new initiatives in postgraduate medical and nursing education.

(2) Empower the patient: let them manage more of their health; extend their choices; respect their time and privacy; let them be the real focus of our service. A unique, seamless package of care for each citizen must be our goal.
(3) Encourage locally generated standards to secure ownership, but do so within a strategic framework.

(4) Search for continuous improvement, not short term effect.

(5) When guarantees are offered make sure they are always delivered - no excuses are acceptable. Guarantees must provide certainty (before contemplating a default on a guarantee sell the boardroom furniture and the chairman's car).

(6) Quality is not an exclusive club; all staff need to share the commitment to it. Treat staff in the way you would like them to treat patients - leaders need to lead by example. 